\title{
La relación entre la habilidad directiva y la satisfacción de los clientes en la industria del mueble de España Un estudio empírico
}

\section{Gonzalo Maldonado}

\section{Guzmán}

Universidad Autónoma de Aguascalientes

gmaldona@correo.uaa.mx

\section{Resumen}

En un mercado altamente competitivo y globalizado como en el que se desarrollan actualmente las organizaciones, las habilidades directivas que poseen los gerentes son un elemento intangible altamente valorado por los empresarios o empleadores; más aún, la satisfacción de los clientes es una de las principales estrategias de los empresarios. Por ello, este trabajo tiene como objetivo analizar los efectos que ejerce la capacidad directiva de los gerentes de logística en la satisfacción de los clientes de las empresas que integran la industria del mueble de España. El análisis empírico se llevó a cabo en una muestra de 322 empresas productoras de muebles con veinte o más trabajadores, y que al menos hubiesen realizado una exportación y/o participado en la Feria Internacional del Mueble de Valencia en las ediciones 2005 y/o 2006. Los resultados obtenidos indican que la experiencia, habilidades y formación ejercen un impacto positivo en el desempeño de los gerentes de logística, quienes a su vez ejercen un impacto positivo en la capacidad directiva, la cual ejerce un impacto positivo en la satisfacción de los clientes.

Keywords: experiencia, habilidades, desempeño, capacidad directiva, satisfacción de los clientes. 


\title{
The relationship between directive ability and customer satisfaction in the Spain furniture industry. An empirical study
}

\begin{abstract}
In a highly competitive and globalized market in which firms are nowadays competing, managers' directive abilities are an intangible element highly valued by employers. Even more, customer satisfaction is one of the main strategies of enterprises. For that reason, this paper aims to analyze the effects of logistics managers' directive abilities on customer satisfaction of the firms that integrate the Spain furniture industry. The empiric analysis was carried out on a sample of 322 furniture manufacturers with twenty or more employees and with at least one export transaction and/or having participated in the Valencia Furniture International Fair, editions 2005 and/or 2006. The results showed that the experience, abilities and formation have a positive impact on the logistics managers' performance, which, in turn, has a positive impact on directive abilities and, ultimately, directive abilities have a positive impact on customer satisfaction.
\end{abstract}

Keywords: experience, abilities, education, performance, directive abilities, customer satisfaction

\section{Introducción}

Las presiones competitivas y los cambios en el ambiente económico han forzado a las empresas a evaluar de nuevo la operación y estructura de sus sistemas de logística. La oscilación de la estrategia de producción local para un mercado local a la de centros nacionales o internacionales de suministro para la manufactura y distribución tienen implicaciones sumamente importantes en las organizaciones, estructura, gerentes, sistemas de control y satisfacción de los clientes (Burcher et al., 2005). Asimismo, la integración virtual de las operaciones de las empresas con sus proveedores de materiales y servicios requiere que los gerentes realicen un monitoreo constante y no un control directo del desempeño de las actividades (Bowersox et al., 2000).

Estos cambios aumentan las habilidades requeridas para los gerentes de logística (Myers et al., 2004; Burcher et al., 2005), especialmente las referentes a los altos ejecutivos dentro de la organización (Poist et al., 2001; Burcher et al., 2005) y al alcance que éstos puedan tener con una buena preparación para afrontar los cambios en el mercado y en la satisfacción de las necesidades de los clientes. Esto tiene un relevante interés, desde hace un par de décadas, en la discusión de las habilidades 
requeridas a los gerentes de logística de Estados Unidos (Murphy y Poist, 1998; Masters y LaLonde, 1998; LaLonde y Pohlen, 2000; Ginter y LaLonde, 2003) y en las habilidades requeridas para la operación de los gerentes en la Unión Europea (Poist et al., 2001; Abdur y Shafreen, 2001; Burcher et al., 2005).

En este sentido, diversos investigadores han estudiado las habilidades, las funciones específicas y el entrenamiento requerido para los gerentes de logística. A pesar de las diferencias en la naturaleza de las operaciones y lo extenso de la función de la logística, las habilidades relevantes de los gerentes tienden a ser similares en la mayoría de las empresas (Richardson, 1991a, 1991b; Abdur y Shafreen, 2001). Así, para tener éxito, los gerentes de logística deben poseer habilidades sociales, de gestión y de solución de problemas (Herron, 1985; Abdur y Shafreen, 2001); conocimiento de las necesidades de los clientes (Buxbaum, 1995; LaLonde y Emmelhainz, 1985; Zineldin, 2004) y habilidades de gestión estratégica (Richardson, 1991a, 1991b; Wanke y Zinn, 2004).

En general, los gerentes de logística necesitan adquirir un amplio rango de habilidades que tendrán que incorporar tanto a sus funciones como al aumento de sus responsabilidades (Poist y Mattingley, 1975; Stock y Lambert, 1987; Poist et al., 2001; Abdur y Shafreen, 2001; Zineldin, 2004); asimismo, poseen pocas habilidades de comunicación, falta de una formación sofisticada en gestión y una inadecuada comprensión de las funciones de marketing; estas carencias son las mayores debilidades de los actuales gerentes de logística (Johnson y Schneider, 1987; Poist et al., 2001; Wanke y Zinn, 2004; Burcher et al., 2005).

A pesar de que las habilidades y competencias requeridas para los gerentes de logística han sido ampliamente analizadas y discutidas en las grandes empresas, y en un contexto de países desarrollados como Estados Unidos y Europa (Murphy y Poist, 1998; Masters y LaLonde, 1998; LaLonde y Pohlen, 2000; Ginter y LaLonde, 2003; Poist et al., 2001; Abdur y Shafreen, 2001; Burcher et al., 2005), son limitados los estudios realizados en la Pyme y, especialmente, en los países en vías de desarrollo (Myers et al., 2004). Por esto, la contribución más importante de la investigación realizada en este estudio es la presentación de los resultados de un análisis de habilidades y competencias de los gerentes de logística en la Pyme de un país en vías de desarrollo (España); para ello, se utiliza una muestra de 322 empresas; de igual forma, otra contribución metodológica por destacar es que se emplean modelos de ecuaciones estructurales para el análisis de los datos. 
El trabajo se ha organizado de la siguiente manera. En el siguiente apartado se revisa el marco teórico y los estudios empíricos previos; después se detallan las características de la investigación empírica; más adelante se analizan los resultados obtenidos y, finalmente, se resumen las principales conclusiones de las que se desprenden, a su vez, las futuras líneas de investigación.

\section{Revisión de la literatura}

Actualmente, en la literatura existe mucho interés por conocer el origen de los gerentes y el camino que éstos han seguido en su profesión; en algunas investigaciones se les ha preguntado sobre detalles demográficos, incluyendo su formación y experiencia en el trabajo (Burcher et al., 2005). Históricamente, los gerentes de logística tienen un nivel medio profesional con un gran conocimiento en la gestión de la cadena de suministro. Pero el interés por la desregulación, la inestabilidad económica, el avance en la tecnología de la información, la globalización de los negocios y el medio ambiente ha invertido esta tendencia (Pooley y Dunn, 1994), en el significado de los cambios en el trabajo, habilidades y responsabilidad de los gerentes de logística (Abdur y Shafreen, 2001).

En este sentido, Murphy y Poist (1991a, 1991b, 1994) consideraron que los gerentes de logística necesitan ser competentes en tres tipos de habilidades: habilidades de negocios, habilidades de logística y habilidades de gestión. En una encuesta que aplicaron estos investigadores a las empresas, a los profesionales y a los académicos de la logística, las habilidades de gestión fueron consideradas como las más importantes de las tres categorías, siguiéndoles las habilidades de logística y en último lugar las habilidades de negocios. El consenso logrado en este estudio es que los entrevistados consideren como primero a los gerentes y después a los profesionales con las competencias y habilidades requeridas, que comprenden tanto las competencias y habilidades generales de gestión como las competencias y habilidades específicas de la logística (Mangan et al., 2001).

Por otro lado, Gammelgaard y Larson (2001) propusieron un modelo de tres factores de habilidades para los gerentes de logística: habilidades básicas de gestión interpersonal, habilidades tecnológicas cuantitativas y habilidades elementales en la gestión de la cadena de suministro. Además, estos autores hicieron énfasis en la importancia de buenas habilidades de comunicación para los actuales gerentes de logística, tanto para comunicarse con sus superiores como con sus subordinados dentro de la organización, así como la existencia de la capacidad para comunicar- 
se, a través de las funciones y las organizaciones, y para una correcta coordinación de la gestión de la cadena de suministro.

El énfasis en las habilidades que los gerentes necesitan poseer para desempeñar sus responsabilidades de manera eficiente parece haber experimentado cambios en las últimas tres décadas. La percepción de la importancia de los sistemas de información de la logística en los años setenta del siglo XX permitió a los gerentes de logística tener un sólido conocimiento en este campo (England y Leenders, 1978). En los años ochenta de ese mismo siglo, el énfasis se cambió a la calidad, la subcontratación internacional, la negociación, la comprensión de los costos y los resultados relacionados a ellos. En los años noventa se inició una oferta de nuevas oportunidades para la industria a través de la formación de nueva y alta tecnología. Todos estos cambios han hecho que la logística contemporánea sea altamente compleja; los nuevos desafíos, la redefinición del papel y el alcance de los gerentes de logística requieren la expansión de sus habilidades y de su experiencia laboral, que es la base en el rápido cambio del medio entorno de los negocios (Abdur y Shafreen, 2001).

Es por ello que Schmidt et al. (1986) consideraron que la experiencia laboral es un elemento esencial en el desempeño y habilidades de los gerentes de logística. Asimismo, McDaniel et al. (1988) concluyeron que la correlación entre la experiencia laboral y el desempeño del trabajo de los gerentes es positiva. Otros trabajos también consideran la importancia que tiene la relación entre la experiencia laboral y el desempeño del trabajo de los gerentes (Blankenship y Taylor, 1938; Taylor y Smith, 1956; Fleishman, 1965; Myers et al., 2004). Por lo tanto, el incremento en el desempeño del trabajo de los gerentes de logística puede ser asociado positivamente con aumentos en sus experiencias de trabajo (Schmidt et al., 1988; Myers et al., 2004).

H1: A mayor nivel de experiencia, mayor será el nivel de desempeño de los gerentes

Varios investigadores han estudiado en Estados Unidos los elementos pertinentes de las habilidades, las funciones específicas de los expertos y el entrenamiento requerido para los gerentes de logística. A pesar de las diferencias en la naturaleza de las operaciones y lo extenso de la función de la logística, las habilidades relevantes para el nivel de los gerentes de logística tienden a ser similares en la mayoría de las empresas (Richardson, 1991a, 1991b; Abdur y Shafreen, 2001). Así, para te- 
ner éxito, los gerentes de logística deben poseer habilidades de funcionalidad, de gestión y solución de problemas (Herron, 1985; Abdur y Shafreen, 2001); conocimiento y entendimiento de las necesidades de los clientes, servicios a los clientes y mantenimiento competitivo de sus experiencias (Buxbaum, 1995; LaLonde y Emmelhainz, 1985; Zineldin, 2004), así como habilidades de gestión estratégica (Richardson, 1991a, 1991b; Wanke y Zinn, 2004).

De igual manera, éstos deben poseer habilidades de comunicación y liderazgo, así como buenos conocimientos de informática (LaLonde y Emmelhainz, 1985; Williams y Currey, 1990; Buxbaum, 1995; Poist et al., 2001; Burcher et al., 2005). Por lo anterior, las habilidades de los gerentes de logística generalmente se asocian con su desempeño (McClelland, 1973; Goleman, 1998; Sandberg, 2000; Myers et $a l ., 2004)$ y son esenciales para el desarrollo de las organizaciones (Hunt y Morgan, 1995).

H2: A mayor nivel de habilidades, mayor será el nivel de desempeño de los gerentes

Por otro lado, los investigadores también se han referido al conocimiento basado en datos, a la gestión y evaluación de flujos monetarios, y a la comprobación de sus éxitos (Williams y Currey, 1990; Abdur y Shafreen, 2001); todo lo anterior con un alto grado de adaptabilidad a las circunstancias propias de los negocios (Gattorna, 1992; Burcher et al., 2005), lo que requiere que los gerentes de logística tengan una buena formación. Asimismo, es importante hacer notar que tanto los atributos personales como los académicos tienen que ser señalados con menor frecuencia que los atributos de las habilidades (Williams y Currey, 1990; Burcher et al., 2005); sin embargo, los gerentes de logística con una alta formación tienden a unir la gran importancia de las técnicas cuantitativas con sus partes menos formadas (Poist y Mattingley, 1975; Abdur y Shafreen, 2001).

En este sentido, la formación de los gerentes de logística repercute de manera significativa en su desempeño laboral, con lo cual las actividades o tareas encomendadas se pueden lograr con mayor facilidad y eficiencia; las modificaciones académicas o de formación de los gerentes de logística comúnmente son consideradas en la literatura como predictores del desempeño laboral (Hunter y Hunter, 1984; Howard, 1986). Por lo tanto, las modificaciones académicas o de formación pueden predecir significativamente el desempeño de la gestión 
tan bien como el desempeño de las habilidades ocupacionales (Singer y Bruhns, 1991; Myers et al., 2004).

H3: A mayor nivel de formación, mayor será el nivel de desempeño de los gerentes

Las características personales representan atributos únicos e individuales de los gerentes de logística como la asertividad y el cuidado de los hábitos personales. Además, los gerentes de logística deben ser creativos, no solamente en la práctica de la gestión con las nuevas técnicas y metodologías, sino también en el conocimiento, la adopción de nuevas formas de pensamiento, percepción, enseñanza del conocimiento y respuesta. Por lo tanto, los futuros gerentes de logística deben tener múltiples capacidades, ser generalistas y capaces de tomar responsabilidades como si fuera un especialista en cada una de las funciones de la logística (Murphy, 1990; Richardson, 1991a, 1991b; Gattorna, 1992; Johnson y Wood, 1993; Gooley, 1994; Pooley y Dunn, 1994; Abdur y Shafreen, 2001; Zineldin, 2004; Burcher et al., 2005), ya que su desempeño afecta significativamente a las metas de los negocios y, por ende, a su capacidad directiva (Daugherty et al., 2000; Myers et al., 2004); por ello, si los gerentes adquieren mayor conocimiento, habilidades, experiencia y formación, las metas de la organización serán más fáciles de lograr, pues la capacidad directiva de los gerentes será mejor (Ireland et al., 2001).

H4: A mayor nivel de desempeño de los gerentes, mayor nivel de su capacidad directiva

Diversos estudios consideran que la satisfacción de los clientes se puede lograr de manera directa (Yi, 1990; Szymanski y Henard, 2001; Spiteri y Dion, 2004; Woo y Ennew, 2004; Russell-Bennett et al., 2007) o indirectamente a través de un eficiente compromiso de gestión de los gerentes (Wetzels et al., 1998; Abdul-Muhmin, 2002, 2005; Hennig-Thurau et al., 2002; Caceres y Paparoidamis, 2007; Cater y Cater, 2008), lo que afecta significativamente la lealtad de los clientes, que es una de las estrategias más importantes de los negocios (Berry y Parasuraman, 1991). Por lo tanto, una excepcional gestión del servicio a los clientes permite obtener buenos beneficios en la satisfacción de los clientes (Hart et al., 1990; McCollough et al., 2000; Holloway y Beatty, 2003; McColl-Kennedy y Sparks, 2003; De Matos et al., 2007; Liao, 2007; Haverila y Naumann, 2010), lealtad e incremento de las ventas (Emerson y Grimm, 1998; Zhang et al., 2005). 
En este sentido, los gerentes de logística deben poseer habilidades eficientes de gestión para planear las actividades de logística para satisfacer las necesidades de sus clientes a través de la contribución que los clientes generan a la rentabilidad de la empresa y de acuerdo con la estrategia empresarial de la organización (Lambert et al., 2008), ya que un buen servicio es importante para lograr una satisfacción de los clientes (Sterling y Lambert, 1989). Como ejemplo de ello está el programa Service Response Logistics (Davis y Manrodt, 1991; Manrodt y Davis, 1992) tendiente a posicionar a las competencias de la logística como las actividades esenciales que facilitan la satisfacción de los clientes (Mentzer et al., 1989; Stalk et al., 1992) por medio de una visión de gestión de los gerentes de logística (Bowersox et al., 2008).

H5: A mayor nivel de habilidades directivas, mayor nivel de satisfacción de los clientes

En la figura 1 se puede apreciar más detalladamente la relación entre las habilidades directivas y la satisfacción de los clientes, así como las hipótesis planteadas en este estudio.

\section{Figura 1}

\section{Modelo conceptual de habilidades directivas}

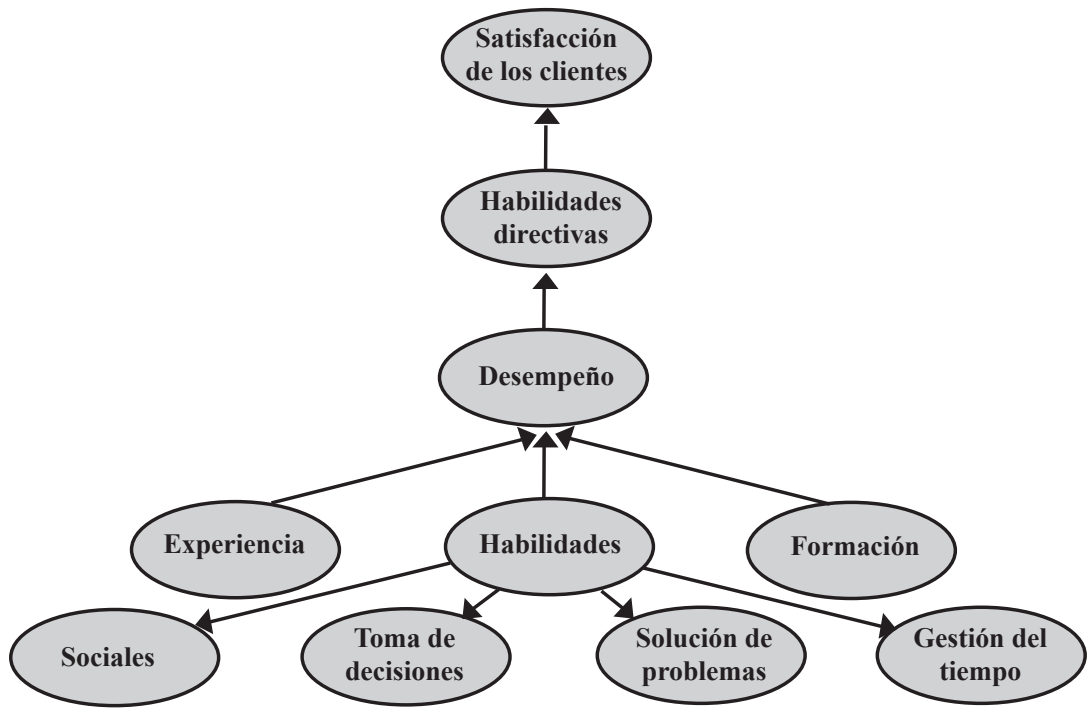

Fuente: Adaptado de Myers et al. (2004) 


\section{Metodología}

Para validar las hipótesis propuestas se llevó a cabo una investigación empírica en las empresas de la industria del mueble de España; para ello, se partió de las siguientes preguntas: ¿por qué seleccionar la industria del mueble y no otra?, ¿qué características particulares reúne la industria del mueble que la hace adecuada para la aplicación de este estudio? La industria del mueble de España reúne cuatro características estructurales esenciales que la hacen adecuada para la aplicación de este tipo de estudios y se centran en las características de su producción, distribución, empleo y comercio exterior. La estructura productiva del sector se caracteriza por una fuerte atomización, pues el grado de concentración de la industria es muy bajo debido a la existencia de numerosas Pyme que en 2007 suponía un $89 \%$ y producía el $38 \%$ de la facturación total. La industria del mueble en España está formada en la actualidad por alrededor de 13170 empresas que dan empleo a 136142 trabajadores con una media de empleados por empresa de 6.4 y con sólo el $10.9 \%$ de las mismas que emplean a 20 o más trabajadores (AIDIMA, 2007).

Por lo que respecta a su distribución geográfica, ésta se concentra en áreas claramente definidas de especialización productiva. El litoral mediterráneo (Cataluña, Comunidad Valenciana y Murcia) es el principal foco nacional productor de muebles. Otros focos importantes son el centro peninsular (Madrid y Toledo), el País Vasco y Navarra, así como la zona central de Andalucía (Sevilla, Jaén y Córdoba). Esta concentración geográfica de la producción de muebles hace específicamente relevante el desarrollo de la función de la logística, tanto de las empresas fabricantes de muebles como de los proveedores de servicios para abastecer tanto al mercado nacional como al internacional.

Por otro lado, el procedimiento que se utilizó en este trabajo de investigación para obtener el marco de referencia consistió en obtener el directorio de las empresas que tenían 20 o más trabajadores, contando para ello con el apoyo de la Asociación Nacional de Industriales y Exportadores de Muebles de España (ANIEME) y de la Feria Internacional del Mueble de Valencia (FIM), con la cual se obtuvo un directorio de 500 empresas, lo que representó un poco más del 38\% del total de la población objeto de estudio (1 300). De igual manera, la encuesta fue enviada por correo ordinario a cada una de las 500 empresas seleccionadas, de las cuales se recibieron 334, y de ellas 12 fueron eliminadas por falta de información, quedando un total de 322 encuestas validadas con un error del $4.8 \%$ y una tasa de respuesta del $53 \%$. 
Como paso previo al análisis de los resultados del trabajo, se llevó a cabo un análisis de fiabilidad y validez de las medidas utilizadas. Las variables utilizadas: habilidades, desempeño, habilidades directivas y satisfacción de los clientes están definidas por escalas unidimensionales. Todos los ítems de estas variables están construidos según una escala tipo Likert de 5 posiciones, con $1=$ completamente en desacuerdo a 5 = completamente de acuerdo como límites.

Las habilidades fueron divididas en: sociales, toma de decisiones, solución de problemas y gestión del tiempo. Las habilidades sociales fueron medidas en una escala de 6 ítems adaptada de Waldman et al. (2001) y Myers et al. (2004); las habilidades en la toma de decisiones fueron medidas en una escala de 6 ítems adaptada de Mintzberg y Westley (2001) y Myers et al. (2004); las habilidades en la solución de problemas fueron medidas en una escala de 4 ítems adaptada de Beyer et al. (1997), Wieringa y Van Bruggen (1997), Ireland et al. (2001) y Myers et al. (2004); y las habilidades en la gestión del tiempo fueron medidas en una escala de 4 ítems adaptada de Carroll y Gillen (1987), Wieringa y Van Bruggen (1997), Barry et al. (1997) y Myers et al. (2004).

La escala para el desempeño fue medida en una escala de 3 ítems y adaptada de Daugherty et al. (2000), Ireland et al. (2001), Zimmerman (2001) y Myers et al. (2004). La habilidad directiva fue medida en 5 dimensiones y adaptada de Daugherty et al. (2000), Ireland et al. (2001), Zimmerman (2001) y Myers et al. (2004). Asimismo, la experiencia fue medida anotando únicamente el número de años que se reportaron y fue adaptada de McDaniel et al. (1988), Schmidt et al. (1988) y Myers et al. (2004). La formación se midió eligiendo, de entre un posible grupo de respuestas, aquella que se adecua a su situación actual y fue adaptada de LaLonde y Pohlen (2000), Ginter y LaLonde (2003) y Myers et al. (2004). Finalmente, la escala para la satisfacción de los clientes fue tomada de Tracey et al. (1999), Koufteros et al. (2002), Stank et al. (2003) y Zhang et al. (2005) con 6 ítems.

Por otro lado, para evaluar la fiabilidad y validez de las escalas de medida se realizó un análisis factorial confirmatorio (AFC) utilizando el método de máxima verosimilitud con el software EQS 6.1 (Bentler, 2005; Brown, 2006; Byrne, 2006). Asimismo, la fiabilidad de las escalas de medida se evaluó a partir del coeficiente $\alpha$ de Cronbach y el índice de fiabilidad compuesta (IFC) (Bagozzi y Yi, 1988). Todos los valores de la escala excedieron el nivel recomendado de 0.70 para el $\alpha$ de Cronbach y el IFC que proporciona una evidencia de fiabilidad y justifica la fiabilidad interna de las escalas (Nunally y Bernstein, 1994; Hair et al., 1995). 
Los resultados de la aplicación del AFC se presentan en el cuadro 1 y sugiere que el modelo de medición proporciona un buen ajuste de los datos $\left(S-B X^{2}=188.9395\right.$; $d f=98 ; p=0.000 ; N F I=0.948 ; N N F I=0.968 ; C F I=0.974 ;$ y $R M S E A=0.054)$. Como evidencia de la validez convergente, los resultados del AFC indican que todos los ítems de los factores relacionados son significativos $(\mathrm{p}<0.001)$ y el tamaño de todas las cargas factoriales estandarizadas son superiores a 0.60 (Bagozzi y Yi, 1988).

\section{Cuadro 1}

\section{Consistencia interna y validez convergente del modelo teórico}

\begin{tabular}{|c|c|c|c|c|c|c|}
\hline Variable & Indicador & $\begin{array}{c}\text { Carga } \\
\text { factorial }\end{array}$ & $\begin{array}{l}\text { Valor-t } \\
\text { robusto }\end{array}$ & $\begin{array}{c}\alpha \text { de } \\
\text { Cronbach }\end{array}$ & IFC & IVE \\
\hline \multirow{4}{*}{$\begin{array}{l}\text { Habilidades de } \\
\text { los gerentes }\end{array}$} & SOS1 & $0.928^{* * * *}$ & $1.000^{\mathrm{a}}$ & \multirow{4}{*}{0.911} & \multirow{4}{*}{0.935} & \multirow{4}{*}{0.783} \\
\hline & DMS2 & $0.948 * * *$ & 34.846 & & & \\
\hline & PSS3 & $0.785^{* * *}$ & 20.458 & & & \\
\hline & TMS4 & $0.870 * * *$ & 26.478 & & & \\
\hline \multirow{3}{*}{ Desempeño } & PE1 & $0.687 * * *$ & $1.000^{\mathrm{a}}$ & \multirow{3}{*}{0.741} & \multirow{3}{*}{0.805} & \multirow{3}{*}{0.579} \\
\hline & PE2 & $0.795 * * *$ & 10.951 & & & \\
\hline & PE3 & $0.797 * * *$ & 12.114 & & & \\
\hline \multirow{3}{*}{$\begin{array}{l}\text { Habilidades } \\
\text { directivas }\end{array}$} & WO1 & $0.690 * * *$ & $1.000^{\mathrm{a}}$ & \multirow{3}{*}{0.743} & \multirow{3}{*}{0.804} & \multirow{3}{*}{0.579} \\
\hline & WO2 & $0.790 * * *$ & 12.773 & & & \\
\hline & WO5 & $0.797 * * *$ & 12.951 & & & \\
\hline \multirow{6}{*}{$\begin{array}{l}\text { Satisfacción } \\
\text { de los clientes }\end{array}$} & $\mathrm{CS} 1$ & $0.775 * * *$ & $1.000^{\mathrm{a}}$ & \multirow{6}{*}{0.888} & \multirow{6}{*}{0.888} & \multirow{6}{*}{0.569} \\
\hline & CS2 & $0.792^{* * * *}$ & 14.994 & & & \\
\hline & $\mathrm{CS} 3$ & $0.730^{* * * *}$ & 11.994 & & & \\
\hline & CS4 & $0.731^{* * *}$ & 13.411 & & & \\
\hline & CS5 & $0.754 * * *$ & 15.425 & & & \\
\hline & CS6 & $0.744 * * *$ & 13.186 & & & \\
\hline \multicolumn{7}{|c|}{$\begin{array}{l}S-B X^{2}(d f=98)=188.9395 ; p=0.000 ; N F I=0.948 ; N N F I=0.968 ; C F I=0.974 ; R M S E A=0.054 \\
\begin{array}{l}a=\text { Parámetros costreñidos a ese valor en el proceso de identificación } \\
* * *=p<0.001\end{array}\end{array}$} \\
\hline
\end{tabular}

El cuadro 1 muestra una alta consistencia interna de los constructos; en cada caso, la $\alpha$ de Cronbach excede el valor de 0.70 recomendado por Nunnally y Bernstein (1994). El IFC representa la varianza extraída entre el grupo de variables observadas y el constructo fundamental (Fornell y Larcker, 1981). Generalmente un IFC superior a 0.60 es considerado como deseable (Bagozzi y Yi, 1988), en este estudio este valor es superior. El índice de la varianza extraída (IVE) fue calculado para cada par de constructos, resultando un IVE superior a 0.50 en todos los factores (Fornell y Larcker, 1981). 
Por lo que respecta a la evidencia de la validez discriminante, la medición se proporciona en dos formas que se pueden apreciar en el cuadro 2. Primero, con un intervalo del 95\% de confidencialidad, ninguno de los elementos individuales de los factores latentes de la matriz de correlación contiene el valor 1.0 (Anderson y Gerbing, 1988); segundo, la varianza extraída entre cada par de constructos es superior que su correspondiente IVE (Fornell y Larcker, 1981). Con base en estos criterios, se puede concluir que las distintas mediciones realizadas en este estudio demuestran suficiente evidencia de fiabilidad y validez convergente y discriminante.

\section{Cuadro 2}

\section{Validez discriminante de la medición del modelo teórico}

\begin{tabular}{l|c|c|c|c}
\hline \multicolumn{1}{c|}{ Variables } & $\mathbf{1}$ & $\mathbf{2}$ & $\mathbf{3}$ & $\mathbf{4}$ \\
\hline $\begin{array}{l}\text { 1. Habilidades de los } \\
\text { gerentes }\end{array}$ & $\mathbf{0 . 7 9 3}$ & 0.066 & 0.083 & 0.085 \\
\hline 2. Desempeño & $0.208,0.308$ & $\mathbf{0 . 5 7 9}$ & 0.074 & 0.061 \\
\hline 3. Habilidades directivas & $0.234,0.337$ & $0.209,0.337$ & $\mathbf{0 . 5 7 9}$ & 0.076 \\
\hline $\begin{array}{l}\text { 4. Satisfacción de los } \\
\text { clientes }\end{array}$ & $0.236,0.348$ & $0.182,0.314$ & $0.209,0.341$ & $\mathbf{0 . 5 6 9}$ \\
\hline
\end{tabular}

La diagonal representa el índice de la varianza extraída (IVE), mientras que por encima de la diagonal se presenta la parte de la varianza (la correlación al cuadrado) Por debajo de la diagonal se presenta la estimación de la correlación de los factores con un intervalo de confianza del $95 \%$.

\section{Resultados}

Se analizó el modelo conceptual propuesto en este estudio utilizando el modelo de ecuaciones estructurales (SEM) con el software EQS 6.1 (Bentler, 2005; Byrne, 2006; Brown, 2006). Asimismo, se realizó un SEM para comprobar la estructura del modelo y obtener los resultados que permitieran contrastar las hipótesis planteadas. La validez nomológica del modelo teórico fue analizada a través del desempeño del test de la Chi cuadrada (Anderson y Gerbing, 1988; Hatcher, 1994). Los resultados obtenidos de este análisis se presentan en el cuadro 3. 


\section{Cuadro 3}

\section{Resultados del SEM del modelo teórico}

\begin{tabular}{|c|c|c|c|c|c|}
\hline Hipótesis & & Relación Estructural & $\begin{array}{c}\text { Coeficiente } \\
\text { Estandarizado }\end{array}$ & $\begin{array}{l}\text { Valor-t } \\
\text { Robusto }\end{array}$ & Medida de los FIT \\
\hline $\begin{array}{l}\text { H1: A mayor nivel de experiencia, mayor nivel } \\
\text { de desempeño de los gerentes. }\end{array}$ & Experiencia & $\longrightarrow$ Desempeño & $0.659^{\star \star \star}$ & 9.038 & $S-B X^{2}{ }_{(127)}=231.0429$ \\
\hline $\begin{array}{l}\text { H2: A mayor nivel de habilidades, mayor nivel } \\
\text { de desempeño de los gerentes. }\end{array}$ & Habilidades & $\longrightarrow$ Desempeño & $0.540^{\star \star \star}$ & 8.133 & $p=0.000$ \\
\hline $\begin{array}{l}\text { H3: A mayor nivel de formación, mayor nivel de } \\
\text { desempeño de los gerentes. }\end{array}$ & Formación & $\longrightarrow$ Desempeño & $0.574^{\star \star \star}$ & 8.338 & $\mathrm{NFI}=0.938$ \\
\hline $\begin{array}{l}\text { H4: A mayor nivel de desempeño, mayores } \\
\text { habilidades directivas de los gerentes. }\end{array}$ & Desempeño & $\longrightarrow$ Habilidad Directiva & $0.728^{\star \star *}$ & 11.197 & \multirow{2}{*}{$\begin{array}{c}\mathrm{NNFI}=0.965 \\
\mathrm{CFI}=0.971 \\
\mathrm{RMSEA}=0.051\end{array}$} \\
\hline $\begin{array}{l}\text { H5: A mayor nivel de habilidades directivas de } \\
\text { los gerentes, mayor satisfacción de los } \\
\text { clientes. }\end{array}$ & Habilidad D. & $\longrightarrow$ Satisfacción de los C. & $0.685^{\star \star \star}$ & 9.773 & \\
\hline
\end{tabular}

Con respecto a la hipótesis $H 1$, los resultados obtenidos $(\beta=0.659, \mathrm{p}<0.001)$ indican que la experiencia tiene efectos significativos en el desempeño de los gerentes; en cuanto a la hipótesis $H 2$, los resultados obtenidos $(\beta=0.540, \mathrm{p}<0.001)$ señalan que las habilidades presentan efectos significativos en el desempeño de los gerentes; en lo referente a la hipótesis $H 3$ los resultados obtenidos $(\beta=0.574, \mathrm{p}$ $<0.001$ ) muestran que la formación posee efectos significativos en el desempeño de los gerentes; por lo que respecta a la hipótesis $H 4$, los resultados obtenidos $(\beta=0.728, \mathrm{p}<0.001)$ indican que el desempeño produce efectos significativos en las habilidades directivas de los gerentes; finalmente, los resultados obtenidos en la hipótesis $H 5(\beta=0.685, \mathrm{p}<0.001)$ señalan que las habilidades directivas tienen efectos significativos en la satisfacción de los clientes. En resumen, se puede comprobar que las cinco variables que se están analizando son significativas $(\mathrm{p}<0.001)$.

\section{Discusión}

Los resultados obtenidos en este trabajo demuestran que la experiencia, las habilidades (sociales, toma de decisiones, solución de problemas y gestión del tiempo) y la formación de los gerentes de logística tienen implicaciones importantes en el desempeño de las actividades gerenciales. Por esta razón, el desarrollo de las habilidades de los gerentes representa un potencial que puede tener una influencia positiva a largo plazo en el logro de las metas y objetivos de las organizaciones. Así, los gerentes necesitan desarrollar aún más sus habilidades para insertarse mejor en 
el área laboral, teniendo a su disposición estudios formales, programas de formación de tipo ejecutivo, observación directa de las habilidades de sus compañeros o de una extensa combinación de estos y otros programas.

En este sentido, la experiencia, las habilidades y la formación de los gerentes son factores importantes que pueden determinar su nivel de desempeño; por lo tanto, los resultados obtenidos en este estudio pueden ser interpretados como requisitos esenciales para determinar el nivel de desempeño de los gerentes de logística de las empresas. Asimismo, la experiencia, las habilidades y la formación de los gerentes representan una diferencia sustancial que puede facilitar el logro de las metas de las organizaciones.

Por otro lado, los gerentes de logística que deseen elegir el uso de programas de entrenamiento para incrementar sus habilidades, después de haber ingresado a la organización, deberán dar respuesta a dos preguntas básicas: ¿qué tipo de entrenamiento es más efectivo de acuerdo con el puesto que desempeña?, y ¿cómo se puede medir el progreso de sus habilidades? (Myers et al., 2004). La evaluación del desempeño formal proporciona una excelente oportunidad para que los gerentes obtengan una retroalimentación verídica de cómo van perfeccionado sus habilidades.

Analizando los trabajos más recientes sobre esta temática, se encuentra que la mayoría de ellos aboga por una integración teórica de la investigación de procesos de los negocios y el rol que juegan los gerentes dentro de las organizaciones. Cada uno de los procesos esenciales de los negocios se subdivide en un largo número de subprocesos (Srivastava et al., 1990; Myers et al., 2004), y la evaluación de los gerentes de logística y la satisfacción de los clientes no son la excepción.

Por lo que se refiere a la satisfacción de los clientes, existen diversos estudios en la literatura actual que presentan a la satisfacción de los clientes como una de las principales metas a las que tiene que aspirar cualquier organización; por ello, la satisfacción de los clientes es considerada como una estrategia empresarial en una mercado cada vez más globalizado y competitivo, pues de ella dependerá, en gran parte, el éxito o fracaso de la organización. En este sentido, los resultados obtenidos en este estudio demuestran que las habilidades directivas de los gerentes de logística son un buen indicador para medir el nivel de satisfacción de los clientes de las empresas. 
En este sentido, una buena gestión de los sistemas de logística deberá acompañarse de buenos resultados en el nivel de satisfacción de los clientes de las empresas, de lo contrario aunque se tengan buenos resultados en la gestión de la logística, pero si no existe un incremento significativo en el nivel de satisfacción de los clientes, los esfuerzos realizados por los gerentes de logística se verán fuertemente afectados; es decir, todos los esfuerzos que realice el personal de la organización deberán tener como fin último la satisfacción de los clientes de las empresas.

Asimismo, el nivel de las habilidades directivas que poseen los gerentes de logística se convierte en un factor esencial para que las organizaciones puedan incrementar el nivel de satisfacción de sus clientes; por esto, los gerentes de logística deberán buscar mecanismos que les permitan mejorar e incrementar sus habilidades directivas porque de ello dependerá que las empresas se beneficien con un incremento significativo en el nivel de satisfacción de sus clientes. Estos mecanismos los tendrá que buscar tanto dentro como fuera de la organización, ya sea por medio de un mejor entrenamiento en la gestión del área de la logística o una formación actualizada en los sistemas de logística empresarial.

De igual forma, los gerentes necesitan involucrarse más en las decisiones que se toman en los sistemas de logística de las organizaciones, pues las decisiones que se tomen afectarán al nivel de satisfacción de los clientes de los negocios; es así que los gerentes deberán buscar nuevas medidas para incrementar el desempeño de las distintas actividades de la logística. Adicionalmente, los diferentes gerentes de logística tienen distintos niveles de experiencia, habilidades y formación y las diversas empresas tienen distintos niveles de satisfacción de sus clientes. Sin embargo, hoy en día, la industria del mueble de España probablemente tiene un gran potencial en el desarrollo y beneficios que conlleva el desarrollo del capital humano en la satisfacción de los clientes.

Cabe aclarar que una limitación de este trabajo es la utilización de un cuestionario enviado por correo normal para la recolección de los datos, que probablemente pueda introducir algunos problemas relacionados con los sesgos de los métodos comunes. Otra limitación es la obtención de la información, puesto que se ha extraído solamente una parte de la información de las habilidades de los gerentes de logística y de la satisfacción de los clientes. Dado el interés por continuar trabajando en esta área trascendental, también es importante señalar que la mayoría de las empresas de la muestra consideran la información requerida como altamente 
confidencial y privada, por lo cual los datos obtenidos no pueden reflejar el desempeño real de los gerentes y la satisfacción real de los clientes.

De igual manera, varias variables fueron medidas usando una escala de tres y cuatro ítems, por lo cual en estudios futuros se deben enfocar en el desarrollo de medidas más comprensivas incrementando el número de ítems. Asimismo, las encuestas fueron dirigidas a los gerentes del área de la logística, por lo cual los resultados pueden diferir cuando se utiliza una población diferente. Es necesario replicar y extender la investigación para obtener una mejor determinación de la escala utilizada; sin embargo, los resultados obtenidos proporcionan importante conocimiento que puede impactar en la práctica habitual de los gerentes de la logística.

En este sentido, es importante ir más allá de los resultados técnicos y discutir: ¿qué efectos se tendrían con la utilización de una formación no tradicional y más tipos específicos de la experiencia y habilidades de los gerentes de logística?; ¿qué resultados se obtendrían si se aplica un modelo más sofisticado para la evaluación de los esfuerzos de los gerentes de logística?; ¿qué actividad específica de la gestión de la logística es la que más impacta en el nivel de satisfacción de los clientes de las empresas? Estas y otras preguntas que puedan surgir se pueden contestar en futuras investigaciones.

\section{Referencias}

Abdul-Muhmin, A. G. (2002). Effects of suppliers' marketing program variables on industrial buyers' relationship satisfaction and commitment. Journal of Business \& Industrial Marketing 17 (7): 63-651.

(2005). Instrumental and interpersonal determinants of relationships satisfaction and commitment in industrial markets. Journal of Business Research 58 (5): 619-628.

Abdur, M.R. y M.B.S. Shafreen (2001). Skill requirements: perception of the senior Asian logistics. International Journal of Physical Distribution \& Logistics Management 31 (5): 374-395.

Anderson, J. y D. Gerbing (1988). Structural equation modeling in practice: a review and recommended two-step approach. Psychological Bulletin 13: 411-423. 
Bagozzi, R.P. y Y. Yi (1988). On the evaluation of structural equation models. Journal of the Academy of Marketing Science 6 (1): 74-94.

Barry, D., C.C. Durnell y J.S. Carroll (1997). Navigating the garbage can: how agendas help managers cope with job realities. Academy of Management Executive 11 (2): 26-42.

Bentler, P. (2005). EQS 6 structural equations program manual, CA: Multivariate Software (www.mvsoft.com).

Berry, L.L. y A. Parasuraman (1991). Marketing services: competing through quality. New York:The Free Press.

Beyer, J.M., P. Chattopadhyay, E. George, W.H. Glick, D.T. Ogilvie y D. Pugliese (1997). The selective perception of managers revisited. Academy of Management Journal 40 (3): 716-737.

Blankenship, A.B. y H.R. Taylor (1938). Prediction of vocational proficiency in three machine operations. Journal of applied psychology 22: 518-526.

Bowersox, D.J., D.J. Closs y T.P. Stank (2000). The mega-trends that will revolutionize supply chain logistics. Journal of bussiness logistics 21 (2): 1-16.

, J.T. Mentzer y T.W. Speh (2008). Logistics leverage. Journal of bussiness strategies 25(2): 85-99.

Brown, T. (2006). Confirmatory factor analysis for applied research. New York: The Guilford Press.

Burcher, P.G., G.L. Lee y A.S. Sohal (2005). A cross country comparison of careers in logistics management in Australia and Britain. International Journal of Logistics Management 16 (2): 205-217.

Buxbaum, P.A. (1995). The next generation of logistics managers: fearless communicators. Transportation and distribution 36 (10): 84-85.

Byrne, B. (2006). Structural equation modeling with EQS, basic concepts, applications, and programming. London: LEA Publishers. 
Caceres, R.C. y N.G. Paparoidamis (2007). Service quality relationship satisfaction, trust, commitment and business-to-business loyalty. Journal of marketing 41 (7,8): 836-867.

Carroll, S.J. y D.J. Gillen (1987). Are the classical management functions useful in describing managerial work? Academy of management review 12(1): $38-51$.

Cater, B. y T. Cater (2008). Relationship-value-based antecedents of customer satisfaction and loyalty in manufacturing. Journal of bussiness \& Industrial marketing 24 (8): 585-597.

Daugherty, P., R.F.Lusch, M.B.Myers y D.A. Griffith (2000). Linking compensation and retention. Supply chain management review 4(3): 64-72.

Davis, Jr., F.W. y K.B. Manrodt (1991). Principles of service response logistics, Proceedings, Chicago: Council of logistics management: 339-355.

De Matos, C.A., J.L. Henrique y C.A.V. Rossi (2007). Service recovery paradox: a meta-analysis. Journal of service research 10 (1): 60-77.

Emerson, C. J. y C. Grimm (1998). The relative importance of logistics and marketing customer service: a strategic perspective. Journal of bussiness logistics 19 (1): 17-32.

England, W.B. y M.R. Leenders (1978). Purchasing and materials management. IL: Richard Irwin.

Fleishman, E.A. (1965). Attitude versus skill factors in work group productivity. Personnel Psychology 18: 253-266.

Fornell, C. y D. Larcker (1981). Evaluating structural equation models with unobservable variables and measurement error. Journal of marketing research 18: 39-50.

Gammelgaard, B. y P. Larson (2001). Logistics skills and competencies for supply chain management. Journal of bussiness logistics 22 (2): 27-50. 
Gattorna, J.L. (1992). Creating an effective logistics system solution: the role of people. International journal of physical distribution \& logistics management 22 (3): 11-14.

Ginter, J.L. y B.J. LaLonde (2003). The Ohio State University 2003 survey of career patterns in logistics. Annual conference proceedings of the council of logistics management, CD ROM.

Goleman, D. (1998). What makes a leader? Harvard bussiness review 76 (6): 93-102.

Gooley, T. (1994). Playing to wing. Traffic management 33 (1): 48-54.

Hair, J.F., R.E. Anderson, R.L. Tatham y W.C. Black (1995). Multivariate data analysis with readings. New York: Prentice-Hall.

Hart, C.W.L., J.L. Heskett y W.E. Sasser (1990). The profitable art of service recovery. Harvard bussiness review 68 (4): 148-156.

Hatcher, L. (1994). A step by step approach to using the SAS system for factor analysis and structural equation modeling. NC: SAS Institute Inc.

Haverila, M. y E. Naumann (2010). Customer complaint behavior and satisfaction in a B2B context: a longitudinal analysis. Journal of service research 10 (2): $45-62$.

Hennig-Thurau, T., K.P. Gwinner y D.D. Gremler (2002). Understanding relationship marketing outcomes: an integration of relational benefits and relational quality. Journal of service research 4 (3): 230-247.

Herron, D.P. (1985). The educational needs of physical distribution managers, in Robertson, J.F. y House, R.G. (Eds), The distribution handbook: 849-855.

Holloway, B.B. y S.E. Beatty (2003). Service failure in online retailing: a recovery opportunity. Journal of service research 6 (1): 92-105.

Howard, A. (1986). College experience y managerial performance. Journal of applied psychology 71 (3): 530-552. 
Hunt, S.D. y R.M. Morgan (1995). The comparative advantage: theory of competition. Journal of marketing 59(2): 1-22.

Hunter, J.E. y R. Hunter (1984). Validity and utility of alternative predictors. Psychological bulletin 96 (1): 72-98.

Ireland, R.D., M.A. Hitt, S.M. Camp y D.L. Sexton (2001). Integrating entrepreneurship and strategic management actions to create firm wealth. Academy of management executive 15 (1): 49-63.

Johnson, J.C. y K.C. Schneider (1987). Marketing managers discuss the strengths and weaknesses of logistical personnel. Logistics and transportation review 23 (4): 325-333.

y D.F. Wood (1993). Contemporary logistics. $5^{\text {th }}$ ed., New York: Macmillan.

Koufteros, X.A., M. Vonderembs y W. Doll (2002). Examining the competitive capabilities of manufacturing firms. Structural equation modeling 9 (2): 256-282

LaLonde, B.J. y L.W. Emmelhainz (1985). Where do you fit in?. Distribution 48 (11): 32-36.

y T.L. Pohlen (2000). The 2000 Ohio State University survey of career patterns in logistics. Annual conference proceedings of the council of logistics management 1: 39-59.

Lambert, D.M., S.J. Garcia-Dastugue y K.L. Croxton (2008). The role of logistics managers in the cross-functional implementation of supply chain management. Journal of bussiness logistics 29 (1): 113-133.

Liao, H. (2007). Do it right this time: the role of employee service recovery performance in customer-perceived justice and customer loyalty after service failures. Journal of applied psychology 92 (2): 475-489. 
Mangan, L., O. Gregory y C. Lalwani (2001). Education, training and the role of logistics managers in Ireland. International journal of logistics: research and applications 4 (3): 313-327.

Manrodt, K.B. y F.W. Davis, Jr. (1993). The evolution to service response logistics. International journal of physical distribution and logistics management 22 (9): 3-10.

Masters, J.M. y B.J. LaLonde (1998). The 1998 Ohio State University survey of career patterns in logistics. Annual conference proceedings of the council of logistics management 1: 35-50.

McClelland, D.C. (1973). Testing for competence rather than for intelligence. American psychologist 1 (1): 1-14.

McColl-Kennedy, J.R. y B.A. Sparks (2003). Application of fairness theory to service failures and service recovery. Journal of service research 5 (3): 251-266.

McCollough, M.A., L.L. Berry y M.S. Yadav (2000). An empirical investigation of customer satisfaction after service failure and recovery. Journal of service research 3 (2): 121-137.

McDaniel, M.A., F.L. Schmidt y J.E. Hunter (1988), Job experience correlates of job performance. Journal of applied psychology 73 (2): 327-330.

Mentzer, J.T., R. Gomes y R.E. Krapfel (1989). Physical distribution service: a fundamental marketing concept. Journal of the academy of marketing science 17: 53-62.

Mintzberg, H. y F. Westley (2001) Decision making: it's not what you think. Sloan management review 42 (3): 89-93.

Murphy, J.V. (1990). Education of transportation managers has changed greatly in past decade. Traffic world: 36-39. 
Murphy, P.R. y R.F. Poist (1991a). Skill requirements of senior-level logistics executives: an empirical assessment. Journal of bussiness logistics 21 (2): 73-94.

(1991b). Skill requirements of senior-level logisticians: practitioner perspectives. International journal of physical distribution \& logistics management 21 (3): 3-14.

(1994). Educational strategies for succeeding in logistics: a comparative analysis. Transportation journal 33: 36-48.

(1998). Skill requirements of senior-level logisticians. International journal of physical distribution \& logistics management 28 (4): 284-301.

Myers, M.B., D.A. Griffith, P.J. Daugherty y R.F. Lusch (2004). Maximizing the human capital equation in logistics: education, experience and skills. Journal of bussiness logistics 25 (1): 21-32.

Nunnally, J.C. e I.H. Bernstein (1994). Psychometric theory. New York: McGraw-Hill.

Poist, R.F. y J.G. Mattingley (1975). Application of quantitative techniques in PDM: a status report. Logistics and transportation review 11 (4): 338-347.

C.A. Scheraga y J. Semeijn (2001). Preparation of logistics managers for the contemporary environment of the European Union. International of physical distribution \& logistics management 31 (7,8): 487-504.

Pooley, J. y S.C. Dunn (1994). A longitudinal study of purchasing positions: 1960-1989. Journal of bussiness logistics 15 (1): 193-212.

Richardson, H.L. (1991a). Career update: logistics in the limelight. Transportation and distribution 32 (1): 46-50.

(1991b). Boost your career with business skills. Transportation and distribution 32 (6): 26-29. 
Russell-Bennett, R., M.K. Janet y L.V. Coote (2007). Involvement, satisfaction, and brand loyalty in small business service setting. Journal of bussiness research 60 (12): 1253-1260.

Sandberg, J. (2000). Understanding human competence at work: an interpretive approach. Academy of management journal 43 (1): 9-25.

Satlk, G., P. Evans y L.E. Shulman (1992). Competing on capabilities: the new rules of corporate strategy. Harvard bussiness review 70: 57-69.

Schmidt, F.L., J.E. Hunter y A.M. Outerbridge (1986). Impact of job experience and ability on job knowledge, work sample performance and supervisory ratings of job performance. Journal of applied psychology 71 (3): 432-439.

y S. Goff (1988). Joint relation of experience and ability with job performance: test of three hypotheses. Journal of applied psychology 73 (1): $46-57$.

Singer, M.S. y C. Bruhns (1991). Relative effect of applicant work experience and academic qualifications on selection interview decisions: a study of betweensample generalizability. Journal of applied psychology 7 (4): 550-559.

Spiteri, J.M. y P.A.Dion (2004). Customer value, overall satisfaction, end-user loyalty, and market performance in detail intensive industries. Industrial marketing management 3 (8): 675-687.

Srivastava, R.K., A.S. Tasadduq y F. Liam (1999). Marketing, business process and shareholder value: an organizationally embedded view of marketing activities and the discipline of marketing. Journal of marketing 62: 168-179.

Stank, T.P., T.J. Goldsby, S. Vickery y K. Savitskie (2003). Logistics service performance: estimating its influence on market share. Journal of bussiness logistics 21 (1): 27-55.

Sterling, J.U. y D.M. Lambert (1989). Customer service research: past, present and future. International journal of physical distribution and logistics management 19 (2): 2-23. 
Stock, J.R. y D.M. Lambert (1987). Strategic logistics management. IL: Irwin.

Szymansky, D.M. y D.H. Henard (2001). Customer satisfaction: a meta-analysis of the empirical evidence. Journal of the academy of marketing science 29 (1): $16-35$.

Taylor, J.G. y P.C. Smith (1956). An investigation of the shape of learning curves for industrial motors tasks. Journal of applied psychology 40 (1): 142-149.

Tracey, M.A., M.A. Vonderembse y J.S. Lim (1999). Manufacturing technology and strategy formulation: keys to enhancing competitiveness and improving performance. Journal of operations management 17 (4): 411-428.

Waldman, D.A., G.G. Ramirez, R.J. House y P. Puranam (2001). Does leadership matter? CEO leadership attributes and profitability under conditions of perceived environmental uncertainty. Academy of management review 44 (1): 134-143.

Wanke, P.F. y W. Zinn (2004). Strategic logistics decision making. International journal of physical distribution \& logistics management 34 (6): 466-478.

Wetzels, M., K. de Ruyter y M. Van Birgelen (1998). Marketing service relationships: the role of commitment. Journal of bussiness \& Industrial marketing $13(4,5): 406-123$.

Wierenga, B. y G.H. Van Bruggen (1997). The integration of marketing problemsolving modes and marketing management support systems. Journal of marketing 63 (1): 23-37.

Williams, A.W. y P. Currey (1990). Desired attributes of logistics managers and a learning hierarchy in management education. Logistics and transportation review 26 (4): 369-379.

Woo, K.-S. y C.T. Ennew (2004). Business-to-business relationship quality: an IMP interaction-based conceptualization and measurement. European journal of marketing 38 (9,10): 1252-1271. 
Yi, Y. (1990. A critical review of consumer satisfaction. En Zeithaml, V.A. (Ed.). Review of marketing. Chicago: American Marketing Association: 68-123.

Zhang, Q., M.A. Vonderembse y J.S. Lim (2005), Logistics flexibility and its impact on customer satisfaction, The international journal of logistics management 16 (1): 71-95.

Zimmerman, E. (2001). What are employees worth? Workforce: 32-36.

Zineldin, M. (2004). Total relationship and logistics management. International journal of physical distribution \& logistics management 34 (3,4): 286-301. (A) 
